\title{
Teil D: Verantwortlichkeiten einer BMB
}

D.1. Bedeutung der Gesellschaftsform einer BMB

D.2. Organisationsverschulden: Haftungsbeschränkung durch Ressortaufteilung im mehrgliedrigen Vereinsvorstand

D.3. Zivilrechtliche bzw. schuldrechtliche Verantwortlichkeit einer BMB 94

D.4. Exkurs: Die Versicherbarkeit der Risiken aus Aufbau und Betrieb einer BMB 



\section{D.1. Bedeutung der Gesellschaftsform einer BMB}

Für die Beantwortung der Grundfrage, wer für Gewährleistung und Haftung einer BMB verantwortlich ist, wird in diesem Abschnitt nach den möglichen Gesellschaftsformen GbR, Verein, GmbH und Stiftung unterschieden. Diese Formen hatten sich in Abschnitt A als meistversprechend für die Ausgestaltung einer BMB erwiesen.

\section{D.1.1. Die Gesellschaft bürgerlichen Rechts (GbR)}

Nach \709 BGB steht den Gesellschaftern einer GbR die Führung der Geschäfte grundsätzlich gemeinschaftlich $\mathrm{zu}$, wobei vertragliche Abweichungen an der Tagesordnung sind. Das bedeutet, dass auch sämtliche Gesellschafter für die Geschäfte verantwortlich sind.

\section{D.1.2. Der eingetragene Verein (e.V.)}

Beim e.V. obliegt es dem Vorstand, die Entscheidungen der Mitgliederversammlung als oberstem Organ des Vereins ( $\mathbb{3} 32$ BGB) auszuführen und nach Außen $\mathrm{zu}$ vertreten. Kurz gesagt ist beim Verein also der Vorstand verantwortlich. Gemäß 』3 $\mathrm{I}$ BGB ist der Verein zum Ersatz aller Schäden verpflichtet, die seine Organe in Ausübung der ihnen zustehenden Verrichtung einem Dritten durch zum Schadenersatz verpflichtende Handlungen zufügen. Grundsätzlich bedeutet dies, dass nicht die Organe für ihre Handlungen zum Schadenersatz herangezogen werden, sondern der Verein, dem jede Möglichkeit der Entlastung (wie z.B. über $\int 8$ I $\mathrm{I}$ BGB) genommen wird und der so gestellt wird, als ob er die zum Schadenersatz verpflichtende Handlung selbst vorgenommen hätte.

Es sind Umstände denkbar, unter denen auch der gesamte Vorstand eines e.V. zur Haftung herangezogen wird. Dies gilt dann unabhängig davon, ob eine Aufgabenteilung zwischen den Vorstandsmitgliedern vereinbart worden war. Eine solche Aufgabenteilung erscheint gerade dann besonders interessant, und zwar für alle einschlägigen Gesellschaftsformen, wenn Wissenschaftler eine Gesellschaft gründen und sich selbst nicht aus dem ihnen vertrauten Bereich der wissenschaftlichen Arbeit herausbewegen wollen. Die Geschäftsführung könnte dann jemand anderem übertragen worden sein. Da es sich hierbei um ein zentrales und hochaktuelles Problem der Organisation von BMB handelt, soll ihm in Abschnitt D.2. mit den von Rechtsprechung und Literatur im Wesentlichen erörterten Schwerpunkten Verein ${ }^{120}$ und $\mathrm{GmbH}$ noch einmal nachgegangen werden. 
Teil D: Verantwortlichkeiten einer BMB

\section{D.1.3. Die Gesellschaft mit beschränkter Haftung (GmbH)}

Die Geschäftsführer einer GmbH müssen nicht selbst Gesellschafter sein. Sie führen die Geschäfte der Gesellschaft und vertreten sie gerichtlich und außergerichtlich im Rahmen einer Gesamtgeschäftsführung, wenn nichts anderes bestimmt ist ( $\mathbb{3} 35 \mathrm{GmbHG}$ ). Die Geschäftführer sind auch für die Einhaltung aller gesetzlichen Vorschriften verantwortlich. Sollte es in Angelegenheiten der Gesellschaft zu einer Verletzung der Sorgfaltspflicht eines ordentlichen Geschäftsmannes kommen, so haften die Geschäftführer gemäß $\mathbb{4} 43$ Abs. 2 GmbHG solidarisch gegenüber der Gesellschaft für den entstandenen Schaden. Wenn Pflichten, die von der GmbH zum Schutz absoluter Rechtsgüter zu beachten sind, auch den Geschäftsführer selbst treffen, kann unter Umständen die Verletzung dieser Pflichten eine deliktische Eigenhaftung auslösen. ${ }^{121}$

\section{D.1.4. Die Stiftung}

Für den Vorstand einer Stiftung gilt im Wesentlichen Vereinsrecht ( $\mathbb{8} 86$ BGB).

\section{D.2. Organisationsverschulden: Haftungsbeschränkung durch Ressortaufteilung im mehrgliedrigen Vereinsvorstand}

Der überwiegende Teil der in Deutschland ansässigen Vereine wird, anders als andere Gesellschaftsformen, von Personen ehrenamtlich neben der normalen beruflichen Tätigkeit geführt. Wegen der Vielfältigkeit und Fülle der zu erledigenden Aufgaben besitzt der Verein deshalb häufig einen mehrgliedrigen Vorstand, der auf den ersten Blick denselben Haftungsrisiken ausgesetzt und demselben Haftungsmaßstab unterworfen scheint wie z.B. die Geschäftsführer einer $\mathrm{GmbH}$. Letztere erhalten aber als Kompensation für das übernommene Haftungsrisiko eine Vergütung.

\section{D.2.1. Stellung des Vorstandes}

Der Vorstand ist ein notwendiges, vom Gesetz in $\int 26$ BGB zwingend vorgeschriebenes Vereinsorgan. Er vertritt den Verein gerichtlich und außergerichtlich in allen Angelegenheiten. Kraft ihrer Amtsstellung sind Vorstände grundsätzlich für alle Angelegenheiten der juristischen Person eines e.V. zuständig. Dieser vom Gesetz vorgesehenen Allzuständigkeit steht zugleich eine umfassende Verantwortung für die Belange des Vereins gegenüber. Dem entsprechend muss ein Vorstand über die notwendigen persönlichen, fachlichen und intellektuellen Fähigkeiten verfügen, um seiner Verantwortung gerecht werden 
D.2. Organisationsverschulden: Haftungsbeschränkung durch Ressortaufteilung im mehrgliedrigen Vereinsvorstand

zu können. ${ }^{122}$ Bei Fehlen dieser Fähigkeiten geht die herrschende Meinung im Haftungsfall von einem Übernahmeverschulden aus, d.h. wer nicht über die erforderlichen Fähigkeiten eines Vorstandes verfügt, darf dieses Amt nicht übernehmen. ${ }^{123}$ Allerdings ist es dem Vorstand gestattet, bestimmte Teile seiner Aufgaben auf Dritte zu übertragen. ${ }^{124}$ In gewissen Situationen sieht das Gesetz sogar die Pflicht vor, einen Dritten bzw. beim mehrgliedrigen Vorstand ein bestimmtes Vorstandsmitglied als Verantwortlichen für eine Aufgabe oder einen Bereich zu bestellen. ${ }^{125}$ Auch unter dem Gesichtspunkt des Organisationsverschuldens ist der Vorstand wegen des Umfanges und der Art seiner Tätigkeiten gut beraten, bestimmte Aufgaben intern zu verteilen oder auf Dritte zu übertragen. ${ }^{126}$ Dadurch stellt sich die Frage, welche Auswirkungen die gesetzlich erzwungene oder freiwillig vorgenommene Aufgaben- bzw. Ressortverteilung auf die persönliche Haftung des einzelnen Vorstandes hat.

\section{D.2.2. Die wichtigsten Haftungsnormen}

Aufgrund der zurzeit immer häufiger eintretenden Liquiditätsschwierigkeiten von Vereinen ist das Risiko der persönlichen Haftung eine reale Bedrohung für Vorstände. Zunehmend versuchen neben Finanzämtern und Sozialversicherungsträgern auch private Dritte, den Vorstand persönlich in die Haftung zu nehmen. Am Markt angebotene Haftpflichtversicherungen zur Absicherung dieses Risikos werden aus Unkenntnis oder Kostengründen in der Regel nicht abgeschlossen. Voraussetzung für die persönliche Haftung des Vorstandes ist allerdings stets, dass ihn aufgrund vorsätzlichen oder (grob) fahrlässigen Verhaltens ein Verschulden an der Pflichtverletzung trifft. In der Regel kommt eine persönliche Haftung des Vorstandes danach nur in Betracht, wenn er persönlich gehandelt hat oder eine aufgrund einer Garantenstellung gebotene Handlung unterlassen hat. ${ }^{127}$ Nur, wenn das nicht handelnde Vorstandsmitglied persönlich positive Kenntnis von den für eine Straftat maßgebenden Tatsachen hatte, kann gegen ihn ein strafrechtlicher Vorwurf erhoben werden. Das gleiche gilt im deliktsrechtlichen Bereich. ${ }^{128}$

\section{D.2.2.1. Haftung wegen Insolvenzverschleppung}

Nach $\int 42$ Abs. 2 S.I BGB hat der Vorstand eines Vereins die Pflicht, rechtzeitig die Eröffnung eines Insolvenzverfahrens zu beantragen, wenn der Verein zahlungsunfähig oder überschuldet ist. Kommt er dieser Pflicht nicht nach, haftet

122 Rüsken in: Klein (2003), Abgabenordnung, $\$ 69$ Rdnr. 55, für das Steuerrecht; Reichert, Handbuch des Vereinsund Verbandsrechts, 9. Aufl., Rdnr. 1930 m.w.Nw.

123 Rüsken (2003) (0. Fn 1), Rdnr. 55; Reichert Rdnr. 1930.

124 Reichert (2003), Rdnr. 1497; Raum in: Wabnitz / Janovsky (2003), Handbuch des Wirtschafts- und Steuerstrafrechts, 2. Aufl., Rdnr. 40 f.; RGZ 157, 235; 162, 166.

125 Zöllner in: Baumbach / Hueck, GmbHG (2000), 17. Aufl., \$ 37 Rdnr. 17 a.

126 Westermann in: Ehrmann (2000), BGB, 10. Aufl., \& 30 Rdnr. 5; BFH/NV 96, 589; RGZ 157, 235; 162, 166.

127 BGH/NJW 96, 1535; Soergel, (o. Fn. 9) Rdnr. 23, Reichert (2003) (0. Fn. 3), Rdnr. 1946; Zöllner (2000) (o. Fn. 4), Rdnr. 57; BGHZ 109, 298 (304).

128 Raum (2003) (o. Fn. 3), Rdnr. 26, 44; Zöllner (2000) (o. Fn. 4) Rdnr. 57. 
der Vorstand den Gläubigern für den Schaden, der aufgrund der Verzögerung des Antrages entsteht. Voraussetzung für die Haftung ist ein Verschulden des Vorstands, d.h. er muss die Zahlungsunfähigkeit oder Überschuldung erkannt haben. In dem Fall regelt $\llbracket 42$ Abs. 2 S. 2 BGB ausdrücklich, dass der Vorstand als Gesamtschuldner haftet. Der Fall der Außenhaftung wegen Insolvenzverschleppung ist der wohl häufigste zivilrechtliche Haftungsfall für einen Vereinsvorstand. ${ }^{229}$

\section{D.2.2.2. Nichterfüllung von Steuerpflichten}

Nach $\int 34$ Abs. I AO haben die gesetzlichen Vertreter einer juristischen Person deren steuerliche Pflichten zu erfüllen. Es handelt sich hierbei um eine gesetzlich normierte öffentlich-rechtliche Pflicht, die nicht auf Dritte übertragen werden kann. ${ }^{130}$ Demgemäß bestimmt $\int 69 \mathrm{AO}$, dass die gesetzlichen Vertreter persönlich haften, soweit Ansprüche aus dem Steuerverhältnis infolge vorsätzlicher oder grob fahrlässiger Verletzung der ihnen auferlegten Pflichten nicht oder nicht rechtzeitig festgesetzt oder erfüllt werden.

\section{D.2.2.3. Nichtabführung von Sozialversicherungsbeiträgen}

Die Abführung von Sozialversicherungsbeiträgen ist ebenso wie die Erfüllung der Steuerpflicht eine gesetzlich normierte Pflicht der juristischen Person. Die Nichtabführung ist eine Straftat nach $\int 266$ a Abs. I StGB. Da der Verein als Arbeitgeber selbst nicht deliktsfähig ist, richtet sich die Strafandrohung gegen sein vertretungsberechtigtes Organ, mithin den Vorstand ( $\int$ I4 Abs. I Nr. I StGB), sofern diesen ein Verschulden trifft. ${ }^{131}$

\section{D.2.2.4. Deliktische Haftung}

Anders als im GmbHG oder im AktG gibt es keine weitere Norm, die die persönliche Haftung des Vereinsvorstandes regelt. Eine Durchgriffshaftung wegen Verletzung der Pflichten als Vorstand wird allgemein abgelehnt. Dieser Anspruch steht allein dem Verein zu. ${ }^{132}$ Der Vorstand kann von Dritten allenfalls auf der Grundlage von Anspruchsnormen des allgemeinen Deliktsrechts (etwa $\iiint 23,826$ BGB) in die Haftung genommen werden. Von besonderer Bedeutung ist in diesem Zusammenhang die Haftung nach $\int 823$ Abs. 2 BGB wegen Verletzung eines Schutzgesetzes, insbesondere wegen strafrechtlich relevanten Verhaltens. ${ }^{133}$

129 Heinrichs in Palandt BGB, 63. Aufl. 2004, $\$ 42$ Rdnr. 3; Ehlers (2002), Haftungsgefahren für die Unternehmensleitung, NWB, Fach 18, S. 3854.

130 Rüsken (2003) (0. Fn. 1), Rdnr. 55 ff.

131 Reichert (2003) (o Fn 3), Rdnr. 1946; Raum (2003) (o Fn 3), Rdnr. 41.

132 Hadding in: Soergel (1987), BGB, 12. Aufl., § 27 Rdnr. 23.

133 Reichert (2003) (o Fn 3), Rdnr. 1940 ff., Ehlers (o. FN 6), S. 3851. 
D.2. Organisationsverschulden: Haftungsbeschränkung durch Ressortaufteilung im mehrgliedrigen Vereinsvorstand

\section{D.2.3. Haftungsbeschränkung durch Ressortaufteilung}

Es ist anerkannte und allgemein übliche Praxis, dass mehrgliedrige Vorstände eine Zuständigkeitsverteilung nach bestimmten Ressorts treffen. Die Aufgaben in einem Verein sind mittlerweile so vielschichtig und umfangreich, dass das einzelne Vorstandsmitglied nicht in der Lage ist, sämtliche Pflichten in einer Person zu erfüllen. ${ }^{134}$ Eine Ressortaufteilung erfolgt in der Regel aufgrund interner, meist mündlicher Absprachen.

\section{D.2.3.1. Deliktische und strafrechtliche Verantwortlichkeit}

Die Ressortaufteilung führt nach herrschender Meinung zu einer Beschränkung der deliktischen und strafrechtlichen Verantwortlichkeit des Vorstandes auf dem Gebiet, für das er nicht zuständig ist. ${ }^{135}$ Der „nicht zuständige“ Vorstand kann sich regelmäßig darauf verlassen, dass der zuständige Vorstand seine Aufgaben ordnungsgemäß erfüllt. ${ }^{136}$ Nur wenn positive Kenntnis besteht, dass die dem Verein obliegenden Aufgaben im Zuständigkeitsbereich des Anderen nicht ordnungsgemäß erfüllt werden, hat der nicht zuständige Vorstand die Pflicht zum Eingreifen. ${ }^{137}$ Teilweise wird zwar die Ansicht vertreten, der Vorstand habe im Falle der Ressortbildung eine Überwachungspflicht in Form regelmäßiger Kontrollen. Eine derartige Pflicht ist indes abzulehnen, da sie die rechtlich zulässige Aufgabenverteilung ad absurdum führen und deren „innerbetrieblichen“ Effekt zunichte machen würde. ${ }^{138}$

\section{D.2.3.2. Verletzung öffentlich-rechtlicher Pflichten}

Hinsichtlich der Erfüllung öffentlich-rechtlicher Pflichten, namentlich der steuerlichen Pflichten, führt eine Ressortaufteilung hingegen nicht zu einem gänzlichen Ausschluss der Verantwortung einzelner Vorstandsmitglieder. Öffentlich-rechtliche Pflichten treffen nach den maßgeblichen Normen stets das Organ als Ganzes. ${ }^{139}$ Nach Ansicht des BFH kann die in $\int 34$ AO normierte Verantwortung des Vereinsvorstandes für die Erfüllung der Steuerpflichten wegen des Grundsatzes der Gesamtverantwortung allenfalls auf eine gewisse Überwachungspflicht beschränkt werden. ${ }^{140}$ Diese haftungsbeschränkende Wirkung tritt aber nur dann ein, wenn die Ressortaufteilung hinreichend bestimmt

134 Reichert (2003) (o Fn. 3), Rdnr. 1485; RGZ 157, 235; 162, 166; Ehlers (2002) (o Fn 6), S. 3851 (für die Unternehmensleitung).

135 Reuter (2001) in: Münchner Kommentar zum BGB, 4. Aufl., \$27, Rdnr. 40; Reichert, Rdnr. 1492 und 1946; BGH, MDR 1997, 151 f.; Ehlers (2002) (0. Rn 6) S. 3856.

136 BGH MDR 1997, 151 f.; Reichert (2003) (o Fn 3), Rdnr. 1932; Raum (2003) (o Fn 1), Rdnr. 41.

137 Reichert (2003) (o Fn. 3), Rdnr. 1946 und 1953; Ehlers (2002) (o Fn 6), S. 3856; Reuter (2001) (o Fn. 14), \& 27 Rdnr. 40; BFH/NV 1998, S. 14 und 60.

138 OLG Hamm, NJW 1971, 817 f.; Westermann (2000) (o Fn 5), \& 27 Rdnr. 7; a.A. Reichert (2003) (o. Fn. 3), Rdnr. 1493, Ehlers (2002) (o Fn. 6), S. 3856.

139 Raum (2003) , (0. Fn. 3) Rdnr. 26; BGH/NJW 94, 2149; BGH/MDR 1997, 151 f.

140 BFH vom 23.06.1998, BStBL. 1998 II, 761 ff; BFH v. 04.05.98; NV 1998, S. 14 und 60; BGH a.a.0., für eine Überwachungspflicht, wenn eine Krisensituation vorliegt; RGZ 98, 98 (100). 
und in schriftlicher Form getroffen wurde..$^{141}$ Die bloß interne, mündlich getroffene Absprache zur Ressortaufteilung ist allenfalls bei der Prüfung eines Verschuldens nach $\mathbb{6} 69$ AO oder im Rahmen der Ermessensausübung der Finanzbehörden nach $\int$ II9 $\mathrm{AO}$ zu berücksichtigen. ${ }^{142}$

Der BFH wendet insoweit beim Vereinsvorstand dieselben Haftungsgrundsätze an wie beim Geschäftsführer einer $\mathrm{GmbH}$. Die Tatsache, dass der Vorstand eines Vereins nicht mit der Sorgfalt eines ordentlichen Kaufmanns tätig sein muss, da er dieses Amt in der Regel ehrenamtlich ausübt, ist nach Auffassung des BFH im Interesse der Sicherstellung der in $\$ 34 \mathrm{AO}$ ausdrücklich normierten steuerlichen Pflichten irrelevant. ${ }^{143}$ Dieser Auffassung ist entgegenzuhalten, dass sowohl das GmbHG als auch das AktG hinsichtlich der Gestaltung der Geschäftsführung wesentlich strengere Formvorschriften aufweisen als das Vereinsrecht. Zum gesetzlichen Auftrag und zu den satzungsmäßigen Rechten des Vereinsvorstandes gehört, sein Zusammenwirken nach pflichtgemäßem Ermessen selbst zu regeln. ${ }^{144}$ Der Vorstand eines Vereins kann daher, anders als die Geschäftsführer einer $\mathrm{GmbH}$, auch ohne ausdrückliche Satzungsermächtigung eine interne Zuständigkeitsverteilung vornehmen, sofern er damit nicht gegen ausdrückliche, anders lautende Satzungsbestimmungen verstößt. ${ }^{145}$

Wenn der Vorstand eines Vereins selbst nicht in der Lage ist, bestimmte Aufgaben wahrzunehmen, so ist er nicht nur berechtigt, sondern sogar verpflichtet, eine besonders kompetente Person für diese Aufgaben zu bestellen..$^{146}$ Kann diese Person mangels entsprechender Regelung in der Satzung nicht als verfassungsmäßiger Vertreter bestellt werden, haftet die juristische Person, d.h. der Verein, aufgrund des Organisationsverschuldens gleichwohl analog $\llbracket 3 \mathrm{I}$ BGB für das Handeln der Person wie für ein Organ bzw. einen verfassungsmäßigen Vertreter, ohne die Möglichkeit der Entlastung nach $\int 83$ I BGB. ${ }^{147}$ Der Vorstand haftet wegen des Verweises auf das Auftragsrecht nach $\ 278$ BGB allerdings nur für ein mögliches Auswahlverschulden, d.h. für ein eigenes Verschulden bei der Übertragung der Aufgabe. Nach der dieser Auffassung zugrunde liegende Ansicht ist ein Beauftragter (d.h. der Vorstand) berechtigt, die Ausführung seines Auftrages einem Dritten zu übertragen, wenn ihm die Übertragung wie im vorliegenden Fall z.B. per Satzung gestattet ist. ${ }^{148}$ Für die Form der Übertragung solch besonderer Aufgaben ist keine bestimmte Form vorgeschrieben. ${ }^{149}$ Der Vorstand haftet daher nicht dafür, dass die Satzung die Möglichkeit der Bestellung als verfassungsmäßiger Vertreter unter Umständen

141 BFH/BStBL. 1998 II, 761 ff; Reichert (2003) (o Fn. 3), Rdnr. 1486 und 1490.

142 BFH/BStBl. 1998 II, $761 \mathrm{ff}$; BFH/NV 1998 S. 14 und 60; BGH/MDR 1997, $151 \mathrm{f}$.

143 BFH/BStBl. 1998 II, $761 \mathrm{ff}$; BFH/NV 1998 S. 14 und 60; BGH/MDR 1997, $151 \mathrm{f}$.

144 Heinrichs (2002) (o Fn. 6), Rdnr. 6; Reuter (2001) (0. Fn. 14) Rdnr. 40.

145 Reuter (2001) (o Fn 14), Rdnr. 40; RGZ 98, 98 (100); a.A. Reichert (2003) (o Fn. 3), Rdnr. 1486, aber einschränkend in 1497.

146 RGZ 157, 235; 162, 166; BFH/NV 1996, 589 (oder er muss sich fremde Hilfe holen).

147 RGZ 157, 235; 162, 166, Westermann (2000) (o Fn 5), \$ 31 Rdnr. 7.

148 Reichert (2003) (o Fn. 3), Rdnr. 1497.

149 Reichert (2003) (o Fn. 3), Rdnr. 1497 RGZ 157, 235; 162, 166. 
D.2. Organisationsverschulden: Haftungsbeschränkung durch Ressortaufteilung im mehrgliedrigen Vereinsvorstand

nicht vorsieht. Er haftet nur für die Erfüllung der Organisationspflicht neben dem Verein. ${ }^{150}$

Das soeben Ausgeführte gilt nach diesseitiger Ansicht auch im Fall der internen Ressortaufteilung eines mehrgliedrigen Vorstands, da diese Aufteilung ebenfalls den Zweck hat, die Aufgaben und Pflichten des Vorstandes bestmöglich zu erfüllen. Der Vorstand kommt demnach mit der internen Ressortaufteilung seinen bestehenden Organisationspflichten nach. ${ }^{151}$ Bei interner Ressortaufteilung haftet das einzelne Vorstandsmitglied neben dem handelnden

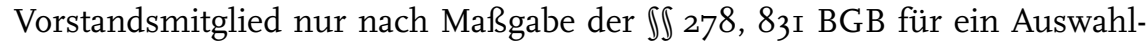
und Übertragungsverschulden bei der Ressortverteilung. Ob der Vorstand bei interner Ressortverteilung nach $\int 6_{9} \mathrm{AO}$ vorsätzlich oder grob fahrlässig gehandelt hat, bestimmt sich nach Ansicht des BFH nach den allgemeinen Grundsätzen. ${ }^{152}$ Ausgehend von den besonderen Umständen des Einzelfalls ist danach zu prüfen, ob der nicht zuständige Vorstand die Pflichtverletzung hätte erkennen können. Dabei sind auch nach Ansicht des BFH die persönlichen Fähigkeiten und Erfahrungen und das nur eingeschränkte persönliche Engagement des ehrenamtlich tätigen Vorstandes zu berücksichtigen. ${ }^{153}$

\section{D.2.4. Fazit}

Die interne mündliche Ressortaufteilung in einem mehrgliedrigen Vereinsvorstand befreit diesen nicht von der Gesamtverantwortung für die Erfüllung der gesetzlich normierten öffentlich-rechtlichen Pflichten. Sie kann aber zu einer Haftungsbeschränkung führen, da sie bei der Prüfung der Schuldfrage in jedem Fall zu berücksichtigen ist. Bei der Verletzung anderer, nicht öffentlichrechtlicher bzw. nicht ausdrücklich gesetzlich normierter Pflichten und Aufgaben des Vorstandes führt die nur mündlich vorgenommene interne Geschäftsverteilung in jedem Fall zu einer Haftungsbeschränkung des nicht zuständigen Vorstandsmitgliedes. Wenn kein Auswahlverschulden vorliegt, kann dieses darauf vertrauen, dass das zuständige Vorstandsmitglied die ihm zugewiesenen Aufgaben ordnungsgemäß erfüllt. Nur wenn positive Kenntnis über Versäumnisse oder Pflichtverletzungen vorliegt, entfällt die haftungsbeschränkende Wirkung. Eine ständige Überwachungspflicht ist nach diesseitiger Ansicht abzulehnen. In jedem Fall empfiehlt es sich, eine entsprechende Haftpflichtversicherung abzuschließen, die die Risiken aus einer Vorstandstätigkeit weitestgehend abdeckt.

150 Die Bestellung eines Vorstandmitglieds als besonderer Vertreter ist von der Rechtsprechung anerkannt. Sauter/Schweiger, Der eingetragene Verein, 16. Aufl. 1997, Rdnr. 313; OLG Hamburg, Rspr. OLG 8, 14.

151 BFH/BStBL. 1998 II, 761 ff; BFH/NV 1998, S. 14 und 60; BGH/MDR 1997, 151. f.; Reichert (2003) (o Fn. 3), Rdnr. 1497.

152 BFH/BStBL. 1998 II, $761 \mathrm{ff}$

153 BFH/BStBl. 1998 II, 761 ff; LG Bonn, NJW-RR 1995, 1435 (erkennt sogar Haftungsentlastung bei schadensträchtiger Tätigkeit an). 
Teil D: Verantwortlichkeiten einer BMB

\section{D.3. Zivilrechtliche bzw. schuldrechtliche Verantwortlichkeit einer BMB}

Neben den Haftungsrisiken stellt sich auch die Frage nach den schuldrechtlichen Risiken und Verantwortlichkeiten einer BMB. Diese Frage lässt sich allerdings nur in Abhängigkeit von den Leistungen einer BMB beantworten, wobei folgende Konstellationen denkbar sind:

- die BMB übernimmt lediglich die Verpflichtung, Biomaterial für Dritte (Klinik, Arzt, Forscher) zu lagern,

- die BMB beschafft selbst Biomaterial, bereitet dieses auf und gibt es an Dritte zur weiteren Verwendung ab,

- die BMB verwahrt Biomaterial für Dritte und gibt es in deren Auftrag an Dritte ab.

Zudem ergeben sich rechtliche Besonderheiten wie z.B. die Anwendung der Vorschriften des HGB, wenn die BMB als Kaufmann anzusehen ist. Dies ist zwingend der Fall, wenn die BMB in der Rechtsform einer AG oder GmbH betrieben wird, da sie dann als Formkaufmann gemäß \6 HGB gilt.

\section{D.3.1. Verwahr- oder Lagervertrag}

Wenn sich die BMB verpflichtet, Biomaterial für Dritte zu lagern, liegt ein Verwahrvertrag nach \ $688 \mathrm{f}$. BGB vor. Die BMB trifft in diesem Fall die Hauptleistungspflicht, die zu verwahrenden Sachen entgegenzunehmen und unterzubringen, d.h. diesen Raum und Obhut zu gewähren. Die BMB muss also zum einen eine geeignete Lagerfläche zur Verfügung stellen. Zum anderen beinhaltet die Pflicht zur Gewährung von Obhut die Pflicht, das Biomaterial vor Zerstörung, Beschädigung, Diebstahl und sonstigen schädigenden Einflüssen zu schützen. Die BMB muss also dafür Sorge tragen, dass die Lagerräume technisch höchsten Anforderungen entsprechen. Sie müssen selbstredend sauber sein, so dass das Biomaterial nicht mit Fremdstoffen kontaminiert werden kann. Eine etwaig notwendige Kühlung muss stets gewährleistet sein, z.B. indem besondere Sicherungsmaßnahmen gegen Stromausfall getroffen werden. Sofern die BMB diese Leistungen als Kaufmann (Formkaufmann, etwa als $\mathrm{GmbH}$ ) anbietet, findet das HGB Anwendung, das in $\iint{ }_{4} 67 \mathrm{ff}$. HGB Spezialvorschriften über das Lagergeschäft als Variante des Verwahrvertrages enthält. Die Besonderheiten des Lagergeschäfts liegen im Wesentlichen darin, dass es stärker formalisiert und der daran anzulegende Sorgfalts- und Haftungsmaßstab wesentlich strenger ist.

\section{D.3.2. Beschaffung und Weitergabe von Biomaterial per Kaufvertrag}

Wenn die BMB selbst Biomaterial gewinnt und daran Dritten Eigentum verschafft, liegt ein Kaufvertrag nach $\iint 433 \mathrm{ff}$. BGB vor. Hauptpflichten der BMB sind in diesem Fall die Lieferung mangelfreien Materials und die rechtskon- 
forme Verschaffung des Eigentums hieran. Mangelfrei im Sinne des $\ 434$ BGB ist das Biomaterial, wenn es „bei Gefahrübergang die vereinbarte Beschaffenheit hat." Wurde keine Beschaffenheit vereinbart, ist das Biomaterial frei von Sachmängeln, wenn es

- sich für die nach dem Vertrag vorausgesetzte Verwendung eignet, oder

- eine Beschaffenheit aufweist, die bei Sachen gleicher Art üblich ist, und die der Käufer nach der Art der Sache erwarten kann.

Einem Sachmangel steht es gleich, wenn eine andere oder eine zu geringe Menge geliefert wurde. Von besonderer Bedeutung ist zudem die Gewährleistung wegen eines Rechtsmangels. Ein Rechtsmangel liegt z.B. vor, wenn das Biomaterial trotz anders lautender Zusage nicht im Eigentum der BMB steht oder einschränkende Persönlichkeitsrechte oder Zweckbestimmungen des Spenders die Nutzbarkeit des Biomaterials einschränken. Es ist eine Nebenpflicht der BMB, den Käufer über solche Mängel aufzuklären. Dies gilt auch dann, wenn die BMB das Material selbst von einem Dritten (Arzt, Klinik etc.) beschafft hat. Sie mag im Falle eines Mangels zwar selbst Regress nehmen können, gegenüber ihrem Kunden und auch gegenüber dem Spender haftet die BMB jedoch selbst ohne Befreiungsmöglichkeit.

Im Zusammenhang mit dem Verkauf von Biomaterialen kommt den Umständen und Bedingungen der Lieferung des Materials besondere Bedeutung zu. Wie jeder andere Verkäufer auch schuldet die BMB als Nebenpflicht grundsätzlich nur dessen Versendung, d.h. die Übergabe des Biomaterials an einen Transporteur (Schickschuld). Der Käufer trägt hierfür in der Regel die Kosten. Nur wenn ausdrücklich vereinbart worden ist, dass die BMB auch den Transport als Hauptleistung zu erbringen hat, schuldet sie diesen (Bringschuld). Die Unterscheidung zwischen beiden Möglichkeiten ist von Bedeutung für die der BMB erwachsenden Gewährleistungspflichten. Sofern die BMB nur die Übergabe des Biomaterials an einen Transporteur schuldet, haftet sie auch nur für die Auswahl von Transporteur (z.B. bei nachgewiesen bekannter Unzuverlässigkeit) und Beförderungsart (z.B. bei mangelhafter Kühlung des Materials). Fehlen die genannten Verschulden, endet die Verantwortlichkeit der BMB mit der Übergabe des Biomaterials an den Transporteur. Hat sie dem Transporteur einwandfreies Biomaterial übergeben, geht die Gefahr des zufälligen Unterganges des Biomaterials (z.B. durch einen Verkehrsunfall des Transporteurs) auf den Erwerber über. Nur wenn die BMB aufgrund entsprechender Vereinbarungen auch den Transport schuldet, geht das Risiko des zufälligen Unterganges erst mit der Übergabe des Materials an den Käufer auf diesen über.

Sofern die BMB Kaufmann ist, finden die Vorschriften des HGB zu ihren Lasten Anwendung. Hierzu zählen insbesondere die Regelungen über den Handelskauf nach $\iint 373$ ff. HGB. Deren Besonderheiten liegen im Wesentlichen in einer Verschärfung der Sorgfaltspflichten und der Haftung für Verzug. Andererseits sind aber auch die Pflichten des Käufers, insbesondere die Rügepflicht, verschärft. Wird das Biomaterial ins Ausland verkauft, finden zudem die Vorschriften des UN-Kaufrechts (CISG) Anwendung, sofern nicht ausdrücklich die Anwendbarkeit eines anderen Rechts, z.B. des deutschen, vereinbart 
Teil D: Verantwortlichkeiten einer BMB

wurde. Auf die diesbezüglichen Besonderheiten einzugehen, würde allerdings den Rahmen des vorliegenden Gutachtens sprengen. ${ }^{154}$

\section{D.3.3. Andere Vertragsgründe für die Abgabe von Biomaterial}

Gibt die BMB Biomaterial an Dritte ab, ohne diesen das Eigentum daran zu verschaffen, scheidet ein Kaufvertrag aus. Gleiches gilt für den Miet- oder Leihvertrag, da statt einer Rückgabe regelmäßig ein Verbrauch des Materials vorgesehen ist. Eine Schenkung kann ebenfalls nicht vorliegen, da auch diese die Eigentumsübertragung voraussetzt. Sofern der BMB ein Nießbrauchrecht an dem Biomaterial zusteht, ist denkbar, dass sie dem Dritten die Ausübung dieses Nießbrauchs nach 』 I059 BGB überlässt. Die Übertragung des Nießbrauchrechts als dingliches Recht ist zwar nicht zulässig, die Überlassung der Ausübung als schuldrechtliche Position ist indes möglich, sofern sie nicht ausgeschlossen wurde. Im Übrigen ist für die Abgabe von Biomaterial auch ein schuldrechtlicher Nutzungsvertrag als Vertrag eigener Art denkbar. Haftung und Gewährleistung der BMB richten sich in allen Fällen nach den getroffenen Vereinbarungen und den gesetzlichen Regelungen des allgemeinen Schuldrechts.

\section{D.3.4. Bearbeitung des Biomaterials und Weitergabe im Auftrag}

Wenn eine BMB das Biomaterial im Auftrag eines Dritten bearbeitet (z.B. Herstellung eines Gewebeschnittes), liegt regelmäßig ein Werkvertrag nach \ 63I ff. BGB vor. Die BMB hat in diesem Fall die Hauptpflicht, entsprechend der Vorgabe des Bestellers ein Werk herzustellen, welches frei von Sach- und Rechtsmängeln ist. Hinsichtlich der Frage, wann ein Mangel vorliegt, kann im Wesentlichen auf Abschnitt D.3.2. verwiesen werden.

Sofern die BMB das Material für Dritte an andere Dritte weitergibt, liegt entweder ein Geschäftsbesorgungsvertrag oder, sofern die BMB ein Kaufmann ist, ein Kommissionsgeschäft nach $\int \mathbb{\int} 383 \mathrm{ff}$. HGB vor. Im Falle eines Kommissionsgeschäfts treffen die BMB besondere Pflichten. So hat sie das Biomaterial unter Umständen selbst zu untersuchen und etwaige Beschädigungen sofort anzuzeigen. Ferner besteht eine Verwahrungspflicht; den Weisungen des Kommittenten (d.h. des abgebenden Dritten) muss Folge geleistet werden.

\section{D.3.5. Fazit}

Je nach der Art der Leistung einer BMB sind eine Vielzahl von Vertragstypen mit unterschiedlichen Haupt- und Nebenpflichten denkbar. Im Mittelpunkt aller Vertragstypen stehen insbesondere die Art und die Qualität respektive der Erhalt des Biomaterials. Die BMB hat entweder dafür Sorge zu tragen, dass das Biomaterial in einwandfreiem Zustand erhalten bleibt (Verwahr- oder 
Kommissionsvertrag), oder sie hat selbst dafür einzustehen, dass das Material eine entsprechende Sach- und Rechtequalität aufweist (Kauf-, Werk-, Nutzungsvertrag). Insoweit ist es ratsam, ein Qualitätssicherungssystem einzurichten und klare Verantwortlichkeiten für bestimmte Arbeitsabläufe zu definieren. Besonderer Wert sollte zudem auf ein gutes Vertrags- und Rechtsmanagement gelegt werden. Die BMB muss sich stets bewusst sein, welche Pflichten ihr aus dem jeweils gewählten Vertragstypus erwachsen, und welche Zusicherungen, Garantien oder Erklärungen von Lieferanten abverlangt werden müssen. Nach diesseitiger Ansicht sind den Lieferanten sogar Standards für die Einwilligungserklärung des Spenders vorzugeben. Trotz all dieser Maßnahmen werden Haftung und Gewährleistung im Außenverhältnis stets alleine die BMB treffen. Den Mitarbeitern einer BMB kann zwar intern in gewissem Umfang die Verantwortung für bestimmte Aufgaben übertragen werden, diese werden aber in der Regel nicht haftbar gemacht werden können, sofern sie nicht vorsätzlich einen Dritten schädigen.

\section{D.4. Exkurs: Die Versicherbarkeit der Risiken aus Aufbau und Betrieb einer BMB}

Auf Veranlassung der Gutachter hat Rüdiger Buyten, Vorstandsmitglied von „Grundeigentümer Versicherungen und Finanzen“ in Hamburg, am 4. Juli 2005 eine Bewertung der Risiken aus Aufbau und Betrieb einer BMB am Beispiel von „Popgen“ in Kiel vorgenommen. Herr Buyten kommt dabei zu folgendem Ergebnis.

\section{D.4.1. Risikobeurteilung}

Die BMB „Popgen“ wird mit Drittmitteln des BMBF gefördert und ist an die Christian-Albrechts Universität zu Kiel respektive das Universitätsklinikum Schleswig-Holstein angegliedert. Versicherungsnehmerin wäre daher die Universität bis zu einer Ausgliederung oder rechtlichen Verselbständigung der BMB. Da die BMB mit Drittmitteln gefördert wird, ist aber auch eine separat einzukaufende Deckung denkbar (Sachdeckungen für Feuer und Betriebsunterbrechung etc. dürften im Rahmen der Universitätsdeckungen bestehen). Im Wesentlichen werden Patienten und Probanden Blutproben vom Klinikpersonal entnommen, aus der dann DNA extrahiert wird. Hierbei bestehende Risiken dürften noch von der Arzthaftpflichtdeckung des Klinikums erfasst sein. Die Aufbereitung und Auswertung erfolgt in einem angeschlossenen Labor durch etwa neun medizinisch-technische Assistentinnen. Die DNA-Proben und teilweise auch Blutproben werden tiefgekühlt in neun Schränken gelagert und zur weiteren Bearbeitung aufbewahrt. Ziel der Untersuchung ist eine genauere Bestimmung genetischer Risiken bei eingegrenzten Bevölkerungsgruppen, die sich zu der Untersuchung meist aufgrund aktueller Befunde bereiterklärt haben. Ich gehe davon aus, dass sich die Frage nach der Versicherbarkeit auf das 
Analyse-, Aufbewahrungs- und Lagerrisiko sowie das Transportrisiko bei Versendung an Drittabnehmer bezieht. Diese Risiken hängen wesentlich von der vertraglichen Gestaltung der Verträge mit Drittunternehmen ab. Hier käme auch eine Haftung in Betracht, wenn die Biomaterialien schadhaft und nicht verwertbar wären.

Bei Verlust oder Verderb der Proben, etwa bei Stromausfall o. ä., wären die Gewinnung und der Wert der Proben als Schaden zu beziffern. Dieser besteht im Wesentlichen in der Wiederbeschaffung der Proben, falls sich die Spender hierzu bereit erklären. Dies dürfte aber mit einem großen finanziellen und organisatorischen Aufwand verbunden sein. Im Wesentlichen wären dann maximal die Drittmittel verloren, und es entstünde ein Imageschaden der Betreiber, die künftig erhebliche Schwierigkeiten bei der Einwerbung weiterer Mittel bekommen könnten. Die Analysemöglichkeiten wären verloren. Datensicherungen bestehen allerdings, so dass die bis dato jeweils erzeugten und anonymisierten Datenmengen erhalten blieben. Lediglich eine Drittverwertung der Proben z.B. durch die Pharmaindustrie wäre dann verloren. Zu erfassen wären auch die verwendeten Geräte und der Wert dieser bei Verlust, soweit keine anderweitige Deckung greift (z. B. Feuer). Ich schätze diesen Bereich auf ca. $€ 300.000$ bis $€ 500.000$.

\section{D.4.2. Versicherungsrechtliche Beurteilung}

Folgende Gefahren sind zunächst betroffen: Feuer und Explosion, Sturm, Leitungswasserschäden im Hause, Einbruchdiebstahl und dadurch verursachte Zerstörung, Sabotage, Energieausfall, Betriebsunterbrechung durch Feuer, Verseuchung. Diese Gefahren können durch die Sachinhaltsversicherung und über die Sachdeckung abgesichert werden, die vielleicht bereits für das Krankenhaus existiert. Hierzu wäre näher zu klären, ob der kommunale Schadenausgleich greift oder eine privatrechtliche Deckung bei einem Versicherer besteht. Unter Umständen besteht im Schadenfall die Schwierigkeit, den Wert der Proben zu ermitteln, da diese nicht unbedingt mit den Betriebskosten und letztlich mit den Drittmitteln übereinstimmen. Die Kosten der einzelnen Proben (Blutentnahme, Extraktion der DNA und Lagerung bzw. Speicherung der gewonnenen Daten) dürfte im Einzelfall nicht erheblich sein. Die Datensicherung müsste zur Risikominimierung in einem entsprechenden Verfahren geprüft werden (Auslagerung in entsprechenden Zeitabständen an getrennten Orten etc.), wie dies für IT-Ressourcen in jedem Betrieb bei Sicherung der existenziellen Daten notwendig ist (Notfallpläne und Risikomanagementsystem).

\section{D.4.3. Konkrete Deckungsnachfrage}

Versicherer aus dem Industriebereich, die sich professionell mit der Deckung der genannten Risiken befassen, haben hier Ablehnung bezüglich des Haftpflichtrisikos signalisiert (konkret der Gerling Konzern, bekanntermaßen hat der HDI aber ebenfalls vergleichbare Annahmekriterien). Dies mag zum einen an dem geringen Umfang der Deckung liegen, hat aber im Wesentlichen seine 
Ursache in den Erfahrungen der Branche aus den Aidsfällen und den typischen Krankenhausrisiken. Die zum Teil erheblichen Untertarifierungen haben zu deutlichen Verlusten in der Haftpflichtsparte dieser Risiken geführt, die nicht durch andere Sparten aufgefangen werden konnten. Im Ergebnis hatte dies eine Kapazitätsverknappung auf dem Sektor zur Folge. Zwar ist das Lagerrisiko zunächst nicht mit dem „Blutrisiko“ verbunden, und die eigentliche Blutentnahme wird noch über die Haftpflichtdeckung des Krankenhauses abgedeckt werden können. Eine Spätfolge aus dem Einsatz und der Verwendung von Gewebeentnahmen und Folgen, die sich aus der Empfehlung einer Datenerhebung ergeben könnten, sind nicht gänzlich ausgeschlossen. Hier kommen auch Personenschäden im weitesten Sinne in Betracht. Zumindest ist dies die Sicht der Haftpflichtversicherer, auch wenn konkrete Schadenbeispiele schwer vorstellbar sind und lediglich die derzeit noch nicht genügend vorstellbare Datenverwendung in der Zukunft betreffen. Risiken dieser Art werden vom Markt daher bei absolutem Bedarf in Form von Pool-Lösungen eingedeckt, da anderweitig keine besseren Kapazitäten zur Verfügung gestellt werden können. Dies erscheint im vorliegenden Fall aber mangels Masse noch nicht probat und praktisch kaum durchführbar. Konkret wäre es daher sinnvoll, mit dem bestehenden Haftpflichtversicherer eine Erweiterung der Deckung für die hier vorhandenen Probleme zu suchen, da dieser ohnehin (einschließlich der Sachdeckung) im Falle eines Schadens mit dem Fall befasst wäre und Abgrenzungsschwierigkeiten hätte. 